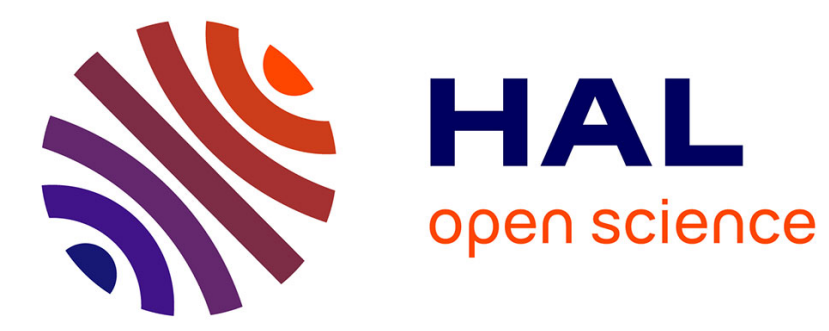

\title{
Minimum feedback vertex set and acyclic coloring
}

Guillaume Fertin, Emmanuel Godard, André Raspaud

\section{To cite this version:}

Guillaume Fertin, Emmanuel Godard, André Raspaud. Minimum feedback vertex set and acyclic coloring. Information Processing Letters, 2002, 84 (3), pp.131-139. hal-00307785

\section{HAL Id: hal-00307785 \\ https://hal.science/hal-00307785}

Submitted on 15 Sep 2009

HAL is a multi-disciplinary open access archive for the deposit and dissemination of scientific research documents, whether they are published or not. The documents may come from teaching and research institutions in France or abroad, or from public or private research centers.
L'archive ouverte pluridisciplinaire HAL, est destinée au dépôt et à la diffusion de documents scientifiques de niveau recherche, publiés ou non, émanant des établissements d'enseignement et de recherche français ou étrangers, des laboratoires publics ou privés. 


\title{
Minimum Feedback Vertex Set and Acyclic Coloring
}

\author{
Guillaume Fertin $^{1}$, Emmanuel Godard $^{2}$, André Raspaud $^{2}$ \\ ${ }^{1}$ IRIN UPRES-EA 2157, Université de Nantes \\ 2 rue de la Houssinière - BP 92208 - F44322 Nantes Cedex 3 \\ ${ }^{2}$ LaBRI U.M.R. 5800, Université Bordeaux 1 \\ 351 Cours de la Libération - F33405 Talence Cedex \\ fertin@irin.univ-nantes.fr, \{godard,raspaud\}@labri.fr
}

\begin{abstract}
In the feedback vertex set problem, the aim is to minimize, in a connected graph $G=$ $(V, E)$, the cardinality of the set $\bar{V}(G) \subseteq V$, whose removal induces an acyclic subgraph. In this paper, we show an interesting relationship between the minimum feedback vertex set problem and the acyclic coloring problem (which consists in coloring vertices of a graph $G$ such that no two colors induce a cycle in $G$ ). Then, using results from acyclic coloring, as well as other techniques, we are able to derive new lower and upper bounds on the cardinality of a minimum feedback vertex set in large families of graphs, such as graphs of maximum degree 3, of maximum degree 4, planar graphs, outerplanar graphs, 1-planar graphs, $k$-trees, etc. Some of these bounds are tight (outerplanar graphs, $k$-trees), all the others differ by a multiplicative constant never exceeding 2 .
\end{abstract}

Keywords: minimum feedback vertex set, acyclic coloring, planar graphs, outerplanar graphs, 1-planar graphs, $k$-trees.

\section{Introduction and Preliminaries}

In this paper, we address the minimum feedback vertex set problem in connected graphs. A feedback vertex set, or $F V S$ of a connected graph $G=(V, E)$ is a subset $V^{\prime}$ of vertices of $G$ such that the (possibly disconnected) graph $G^{\prime}$ induced by $V \backslash V^{\prime}$ is a forest (that is, contains no cycle). A minimum feedback vertex set, or MFVS in $G$ is a $F V S$ of minimum cardinality, and is denoted $\bar{V}(G)$. The MFVS problem finds its motivation in several areas of computer science such as combinatorial circuit design [12], monopolies in distributed networks [17] or placement of converters in optical networks $[14,19]$.

It has been shown that the problem of finding a MFVS in a graph $G$ is NP-hard in general ; however, a large literature shows that it becomes polynomial when addressed to specific families of graphs such as interval graphs [15], permutation graphs [7] and co-comparability graphs [10], among others. Moroever, several recent papers have developed methods to find bounds on the minimum feedback vertex set in several families of graphs, such as $d$-dimensional grids and tori, butterfly networks and hypercubes [2, 9, 16, 12]. For general graphs, the best known algorithm has an approximation ratio of $2[1]$. We refer to [11] for a rather complete and recent survey on the feedback vertex set problem.

In this paper, we establish an interesting connection between this problem and a coloring 
problem on graphs, acyclic coloring. An acyclic coloring of a graph $G=(V, E)$ is a coloring of its vertices, satisfying the two following rules :

(a) No two neighboring vertices are assigned the same color (this is also denoted as proper coloring).

(b) Let $V_{a} \subseteq V$ be the set of vertices of $G$ that are assigned color $a$. Then, for any $a \neq b$, the subgraph $G^{\prime \prime}$ of $G$ induced by $V_{a} \cup V_{b}$ must be acyclic.

The minimum number of colors necessary to color $G$ is called acyclic chromatic number of $G$, and is denoted $a(G)$. Similarly, for a family $\mathcal{F}$ of graphs, the acyclic chromatic number of $\mathcal{F}$, denoted by $a(\mathcal{F})$, is defined as the maximum $a(G)$ over all graphs $G \in \mathcal{F}$. Determining the acyclic chromatic number of a graph $G$ is also a NP-complete problem in general. It has been widely studied in the past 25 years, and in particular, as we will see in the following, several authors have been able to determine $a(\mathcal{F})$ for several families $\mathcal{F}$ of graphs such as graphs of maximum degree 3 [13], of maximum degree 4 [8], planar graphs [3], planar graphs with "large" girth [5], outerplanar graphs (see for instance [18]), 1-planar graphs [4], etc.

Our main contribution here is to establish a direct connection between the acyclic chromatic number of a connected graph $G$ and the cardinality of a minimum feedback vertex set in $G$. Starting from this, we will develop some techniques to determine lower and upper bounds on the MFVS cardinality of several large families of graphs, such as graphs of maximum degree 3 , of maximum degree 4, planar graphs, planar graphs with girth greater than or equal to 5 (resp. 7), outerplanar graphs, 1-planar graphs, $k$-trees, etc. In some of these cases, the given bounds are tight ; in all the other cases, we are able to show that the lower and upper bounds differ by a multiplicative constant at most equal to 2 . Most of the time, this constant will be strictly less than 2 . These upper bounds might not always be tight, but they have the very interesting property that they apply in very large families of graphs (consider planar graphs or graphs of maximum degree $d \in\{3,4\}$, for instance). Until now, close bounds on the cardinality of a MFVS were given in much smaller families (e.g. $d$-dimensional grids, butterfly networks, hypercubes).

In section 2, we will first formalize the connections that exist between acyclic chromatic number and cardinality of a MFVS. In section 3 we will then determine lower and upper bounds on the MFVS cardinality of the above mentioned families of graphs.

\section{Connection between MFVS and acyclic coloring}

In this section, we first make the connection between the acyclic chromatic number of a graph $G$ and the cardinality of a MFVS in $G$. Our results are the following.

Lemma 1 Let $G=(V, E)$ be a graph of order $|V|=N$. If $a(G) \leq k$, then $|\bar{V}(G)| \leq \frac{k-2}{k} \cdot N$.

Proof : Suppose we have an acyclic coloring of $G=(V, E)$ that uses $k$ colors. We then partition the set $V$ in $k$ classes $V_{1}, V_{2}, \ldots V_{k}$, according to the colors given to the vertices of $G$. In that case, by definition of acyclic coloring, for any $1 \leq i<j \leq k, V_{i} \cup V_{j}$ induces a subgraph of $G$ that is acyclic.

Now let $s_{i, j}=\left|V_{i}\right|+\left|V_{j}\right|$ for all $1 \leq i<j \leq k$. We then have $\sum_{1 \leq i<j \leq k} s_{i, j}=(k-1) N$, because each $\left|V_{i}\right|$ appears $k-1$ times in this sum. However, there are $A=\frac{k(k-1)}{2}$ terms $s_{i, j}$ in this sum. Thus, there exists a pair $\left(i_{0}, j_{0}\right)$ such that $s_{i_{0}, j_{0}} \geq \frac{(k-1) N}{A}$, that is such that $s_{i_{0}, j_{0}} \geq \frac{2 N}{k}$. Let $V_{0}=V_{i_{0}} \cup V_{j_{0}}$; in that case, $V-V_{0}$ is a FVS and its cardinality is less than or equal to $N-\frac{2 N}{k}$, that is to $\frac{k-2}{k} N$.

Note that there exists an infinite number of cases for which this bound is tight, see Section 3.5. 
We also have a simple lower bound for $|\bar{V}(G)|$.

Lemma 2 For any non trivial graph $G,|\bar{V}(G)| \geq a(G)-2$.

Proof : Let $\bar{V}(G)$ be a feedback vertex set of $G$. We define the following coloring : each vertex of $\bar{V}(G)$ is assigned a different color. The remaining vertices of $V \backslash \bar{V}(G)$ (which induce a forest) are colored by 2 new colors. This coloring is clearly proper and acyclic, and thus $a(G) \leq|\bar{V}(G)|+2$.

We note that the bound given in Lemma 2 is also tight, see the MFVS cardinality of complete graphs or complete $k$-partite graphs (cf. Tables 1 and 2).

Caragiannis et al. [9] proved a general lower bound for the MFVS cardinality of graphs of maximum degree $r$. Their result, that we will use several times in the rest of this paper, is the following.

Lemma 3 [9] Any feedback vertex set $\bar{V}(G)$ in a graph $G=(V, E)$ with maximum degree $r$ satisfies : $|\bar{V}(G)| \geq \frac{|E|-|V|+1}{r-1}$.

\section{Applications}

Formally, the MFVS cardinality of a family $\mathcal{F}$ of graphs is defined as the maximum cardinality of a MFVS over all the graphs that belong to $\mathcal{F}$. Let $\bar{V}(\mathcal{F})$ denote this value. Using the result of Lemma 1 , it is now possible to exploit already known results about acyclic coloring in several families of graphs, and incorporate them in Lemma 1 to get upper bounds results. Moreover, using several different techniques, we will also prove lower bound results. Namely, we define by lower bound on the MFVS cardinality of $\mathcal{F}$ any lower bound for $\bar{V}(\mathcal{F})$.

In some cases, those lower and upper bounds coincide, up to a small additive constant (outerplanar graphs, $k$-trees), showing that Lemma 1 is tight ; in other cases, our lower bounds do not meet the upper bound, but they differ by a multiplicative constant $c$ never exceeding 2. Most of the time, $c$ will be strictly less than 2 .

\subsection{Graphs of Maximum degree 3}

Theorem 1 [13] For any graph $G$ with maximum degree $3, a(G) \leq 4$.

Applying Lemma 1 to the above result immediately gives the following proposition.

Proposition 2 (MFVS in Graphs of Maximum Degree 3 - Upper bound) For any graph $G$ of maximum degree 3 and order $N,|\bar{V}(G)| \leq \frac{N}{2}$.

Proposition 3 (MFVS in Graphs of Maximum Degree 3 - Lower bound) For any integer $N \geq 3$, there exists a graph $G$ of maximum degree 3 and of order $N$, such that $|\bar{V}(G)|=\left\lfloor\frac{N}{3}\right\rfloor$.

Proof: Let $N=3 p+q, 0 \leq q \leq 2$. In that case, construct a cycle of length $2 p+q, C_{2 p+q}$, with vertices $u_{1}, u_{2}, \ldots, u_{2 p+q}$. Now add a vertex $v_{i}$ for every $1 \leq i \leq p$, and the edges $\left(v_{i}, u_{2 i-1}\right)$ and $\left(v_{i}, u_{2 i}\right)$. The new graph $G$ is clearly of maximum degree 3. Moreover, $|\bar{V}(G)| \geq p$ because there are $p$ edge disjoint $K_{3}$ in $G$ (those induced by vertices $u_{2 i-1}, u_{2 i}$ and $v_{i}(1 \leq i \leq p)$ ). We have in fact $|\bar{V}(G)|=p$ because removing vertex $u_{2 i-1}, 1 \leq i \leq p$ and their incident edges will result in a forest, that is an acyclic graph. 


\subsection{Graphs of Maximum degree 4}

Theorem 4 [8](also independently shown by Kostochka) For any graph $G$ with maximum degree $4, a(G) \leq 5$.

Applying Lemma 1 to this result immediately gives the following proposition.

Proposition 5 (MFVS in Graphs of Maximum Degree 4 - Upper bound) For any graph $G$ of maximum degree 4 and of order $N,|\bar{V}(G)| \leq \frac{3 N}{5}$.

Proposition 6 (MFVS in Graphs of Maximum Degree 4 - Lower bound) For any integer $N \geq 4$, there exists a graph $G$ of maximum degree 4 and of order $N$, such that $|\bar{V}(G)| \geq 2 \cdot\left\lfloor\frac{N}{4}\right\rfloor$.

Proof : We will use 2 different "basic" graphs, that we will arrange together to form graphs $G$ of maximum degree 4 and order $N$, that satisfy the given inequality. Those two basic graphs are the complete graphs $K_{3}$ and $K_{4}$. We note that for any $n \geq 3,\left|\bar{V}\left(K_{n}\right)\right|=n-2$ (indeed, deleting a vertex in $K_{n}$ and its incident edges yields $K_{n-1}$, which is acyclic iff $n-1=2$ ). In particular, $\left|\bar{V}\left(K_{4}\right)\right|=2$ and $\left|\bar{V}\left(K_{3}\right)\right|=1$.

Suppose now that $N=4 p$. In this case, construct $G$ by taking $p$ copies of $K_{4}$ (call them the $\left.K_{4, i}, 1 \leq i \leq p\right)$, and connect one vertex of $K_{4, i}$ to a vertex of $K_{4, i+1}$ by an edge $e_{i}$, for every $1 \leq i \leq p-1$, in such a way that the resulting graph remains of maximum degree 4 (this is always possible : it suffices that any vertex in $K_{4, i}$ be involved in at most one connection to another $K_{4, j}$, $j \in\{i-1, i+1\})$. The resulting graph $G$ is of order $N=4 p$, and clearly we have $|\bar{V}(G)| \geq 2 p$, because no edge $e_{i}$ participates in a cycle in $G$, and for every $K_{4, i}$, two vertices at least must be removed in order to get an acyclic graph. When $N=4 p+1$ (resp. $N=4 p+2$ ) add one (resp. two) pendent edge(s) to $G$. In that case, $|\bar{V}(G)| \geq 2 p$. Finally, when $N=4 p+3$, we take a copy of $K_{3}$ that we add to the original "chain of $K_{4}$ " by connecting any vertex $v$ of $G$ such that $\operatorname{deg}(v)=3$ to any vertex of $K_{3}$. In that case, $\left|\bar{V}\left(K_{3}\right)\right|=1$, and thus one more vertex is to be removed in order to get an acyclic graph. Thus $|\bar{V}(G)| \geq 2 p+1$. Globally, this gives the result. $\square$

\subsection{Planar Graphs}

Borodin [3] has shown that for any planar graph $G, a(G) \leq 5$. He also showed an example of a planar graph for which acyclic coloring needs 5 colors (cf. graph $G_{2}$ of Figure 1), thus obtaining optimality, and showing that if $\mathcal{P}$ denotes the family of planar graphs, then $a(\mathcal{P})=5$. Combining this deep result with the one of Lemma 1, we get the following proposition.

Proposition 7 (MFVS in Planar Graphs - Upper bound) For any planar graph $G$ of or$\operatorname{der} N,|\bar{V}(G)| \leq \frac{3 N}{5}$.

We are also able to show a lower bound of $|\bar{V}(G)| \geq\left\lfloor\frac{N}{2}\right\rfloor$ for planar graphs of arbitrary order $N$, thus showing that the multiplicative ratio between the upper and lower bound is equal to 1.2. This is the purpose of the following proposition.

Proposition 8 (MFVS in Planar Graphs - Lower bound) For any integer $N \geq 3$, there exists a planar graph $G$ of order $N$, such that $|\bar{V}(G)| \geq\left\lfloor\frac{N}{2}\right\rfloor$.

Proof : We will use 4 different "basic" graphs, that we will arrange together to form graphs $G$ of greater order $N$, that satisfy $|\bar{V}(G)| \geq\left\lfloor\frac{N}{2}\right\rfloor$. We will first detail the arguments for 4 basic graphs : they are respectively $K_{3}, K_{4}$, and the graphs $G_{1}$ and $G_{2}$ of Figure 1 . As seen in proof of Proposition 6 , in the cases $n=3$ and $n=4$, we have respectively $\left|\bar{V}\left(K_{3}\right)\right|=1$ and $\left|\bar{V}\left(K_{4}\right)\right|=2$. We also note that $K_{3}$ and $K_{4}$ are both planar. We now consider $G_{1}$. It is planar of order 5 , and $\left|\bar{V}\left(G_{1}\right)\right|=2$. Indeed, removing one of the 5 vertices is not sufficient to get an acyclic graph, while removing both vertices $v_{1}$ and $u$ is. Finally, let us consider $G_{2}$, planar and of order 6 . We have $\left|\bar{V}\left(G_{2}\right)\right|=3$. It has been shown that $a(G)=5[3]$. Thus we have $\left|\bar{V}\left(G_{2}\right)\right| \geq 3$ by Lemma 2 , and actually we have $\left|\bar{V}\left(G_{2}\right)\right|=3$ by Lemma 1 . 

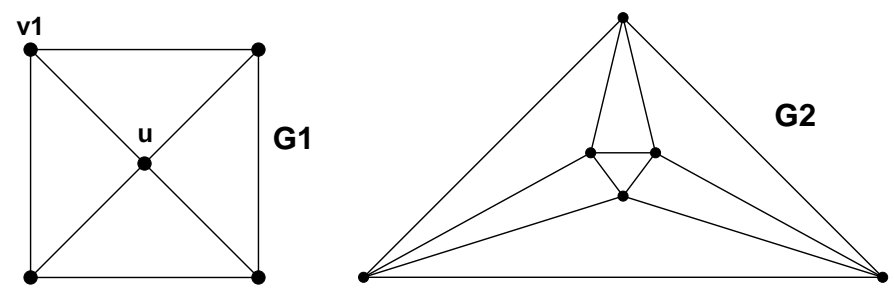

Figure 1: Graphs $G_{1}$ and $G_{2}$

Now that we have determined the cardinality of a MFVS for our 4 basic graphs, we will use these graphs to construct graphs $G$ of arbitrary order $N \geq 3$ such that $|\bar{V}(G)| \geq\left\lfloor\frac{N}{2}\right\rfloor$. For this, we distinguish four cases :

- $N=4 p, p \geq 1$. As in proof of Proposition 6 , we construct $G$ by taking $p$ copies of $K_{4}$ (call them the $\left.\bar{K}_{4, i}, 1 \leq i \leq p\right)$, and we connect one vertex of $K_{4, i}$ to a vertex of $K_{4, i+1}$ by an edge $e_{i}$, for every $1 \leq i \leq p-1$. Here, we do it in such a way that the resulting graph remains planar (which is always possible). The resulting graph $G$ is of order $N=4 p$, and for the same reasons as in proof of Proposition 6, we have $|\bar{V}(G)| \geq 2 p$.

- $N=4 p+1$. Do the same, taking $p-1$ copies of $K_{4}$ and a copy of $G_{1}$. We then see that $|\bar{V}(G)| \geq 2(p-1)+2$.

- $N=4 p+2$. Do the same, taking $p-1$ copies of $K_{4}$ and a copy of $G_{2}$. We then see that $|\bar{V}(G)| \geq 2(p-1)+3$.

- $N=4 p+3$. Do the same, taking $p$ copies of $K_{4}$ and a copy of $K_{3}$. We then see that $|\bar{V}(G)| \geq 2 p+1$.

In all the cases, we have shown that it is possible to find a planar graph $G$ of arbitrary order $N \geq 2$ such that $|\bar{V}(G)| \geq\left\lfloor\frac{N}{2}\right\rfloor$, which proves the above proposition.

For any graph $G$, the girth of $G$, denoted by $g$, is the minimum chordless cycle in $G$. In [5], the authors have proved the following theorem.

Theorem 9 [5] For any planar graph $G$ with girth $g$ :

(9a) If $g \geq 5$, then $a(G) \leq 4$.

(9b) If $g \geq 7$, then $a(G) \leq 3$.

Using the above result and the one of Lemma 1, we get the following result.

Proposition 10 (MFVS in Planar Graphs with girth $\boldsymbol{g}$ - Upper bound) For any planar graph $G$ of order $N$ and girth $g$ :

(10a) If $g \geq 5$, then $|\bar{V}(G)| \leq \frac{N}{2}$.

(10b) If $g \geq 7$, then $|\bar{V}(G)| \leq \frac{N}{3}$.

Similarly, we are able to derive lower bounds for the size of a MFVS in a graph $G$ of arbitrary order $N, G$ belonging to the family of planar graphs with girth at least 5 (resp. with girth at least 7). This is the purpose of Propositions 11 and 12 below.

Proposition 11 (MFVS in Planar Graphs of Girth $\geq 5$ - Lower bound) For any integer $N \geq 20$, there exists a planar graph $G$ of girth 5 and of order $N$ such that $|\bar{V}(G)| \geq \frac{3 N}{10}-2$. 
Proof : Starting from the dodecahedron graph $H$ of Figure 2, we will construct a graph $G^{\prime}$ satisfying the above property. In order to prove this, we will use an argument similar to the one of Proof of Proposition 8. $H$ is planar and of girth 5. Moreover, it has 20 vertices, 30 edges and is of maximum degree 3 , thus by Lemma 3 , we have $|\bar{V}(H)| \geq \frac{11}{2}$. In other words, $|\bar{V}(H)| \geq 6$, and it can be seen that the equality holds.

We will also use 3 other graphs for our construction : the cycle of length $5, C_{5}$, and graphs $G_{5}$ and $G_{5}^{\prime}$ of Figure 3(left and middle). Those three graphs are planar and of girth 5 and, by Lemma 3 , it is easy to check that $\left|\bar{V}\left(C_{5}\right)\right|=1,\left|\bar{V}\left(G_{5}\right)\right| \geq 3$ and $\left|\bar{V}\left(G_{5}\right)\right| \geq 2$.

Now, for any $N=20 p+q, p \geq 1$ and $0 \leq q \leq 19$, construct a chain of $p$ copies of $H$, to which we add : 1) a path of order $q$ if $0 \leq q \leq 4 ; 2)$ a copy of $C_{5}$ plus a path of order $q-5$ if $5 \leq q \leq 7 ; 3)$ graph $G_{5}^{\prime}$ of Figure 3 (middle) plus a path of order $q-8$ if $8 \leq q \leq 11 ; 4$ ) graph $G_{5}$ of Figure 3(left) plus a path of order $q-12$ if $12 \leq q \leq 16 ; 5)$ graph $G_{5}$ of Figure 3(left) plus a copy of $C_{5}$ plus a path of order $q-17$ if $17 \leq q \leq 19$. Call the resulting graph $G^{\prime}$.

Clearly, $G^{\prime}$ can be constructed in such a way that it is planar. Moreover, it is of girth 5 , and it can be seen that :

- $\left|\bar{V}\left(G^{\prime}\right)\right| \geq \frac{3(N-q)}{10}$ if $0 \leq q \leq 4$;

- $\left|\bar{V}\left(G^{\prime}\right)\right| \geq \frac{3(N-q)}{10}+1$ if $5 \leq q \leq 7$;

- $\left|\bar{V}\left(G^{\prime}\right)\right| \geq \frac{3(N-q)}{10}+2$ if $8 \leq q \leq 11$;

- $\left|\bar{V}\left(G^{\prime}\right)\right| \geq \frac{3(N-q)}{10}+3$ if $12 \leq q \leq 16$;

- $\left|\bar{V}\left(G^{\prime}\right)\right| \geq \frac{3(N-q)}{10}+4$ if $17 \leq q \leq 19$.

In all the cases, it can be seen that $\left|\bar{V}\left(G^{\prime}\right)\right| \geq \frac{3 N}{10}-2$.

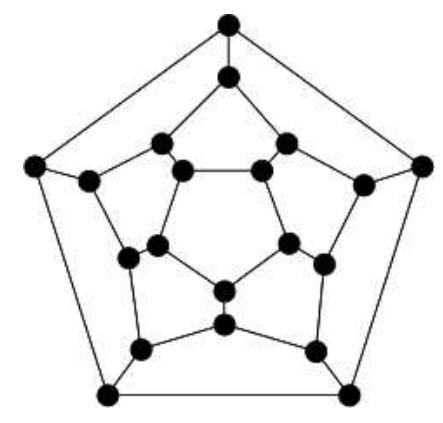

Figure 2: The dodecahedron graph $H$
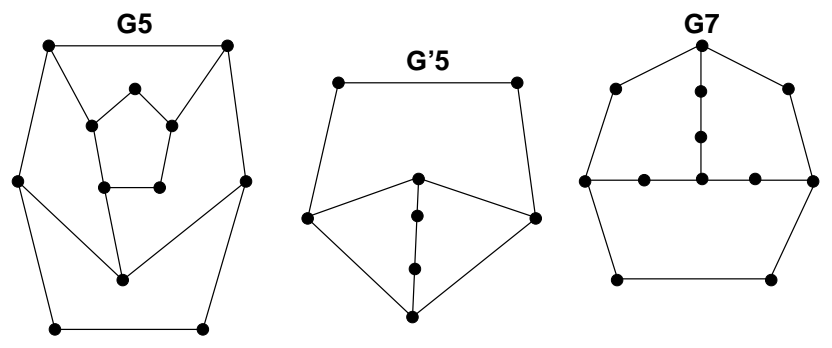

Figure 3: Graphs $G_{5}, G_{5}^{\prime}$ and $G_{7}$ 
Proposition 12 (MFVS in Planar Graphs of Girth $\geq 7$ - Lower bound) For any integer $N \geq 12$, there exists a planar graph $G$ of girth 7 and of order $N$ such that $|\bar{V}(G)| \geq \frac{N}{6}-1$.

Proof : Similarly to Proof of Proposition 11, using graph $G_{7}$ of Figure 3(right) as our "basic graph". $G_{7}$ has 12 vertices, 14 edges, and is of maximum degree 3, thus by Lemma 3 , we have $\left|\bar{V}\left(G_{7}\right)\right| \geq \frac{3}{2}$. In other words, $\left|\bar{V}\left(G_{7}\right)\right| \geq 2$, and it can be seen that the equality holds. Now, for any $N=12 p+q, p \geq 1$ and $0 \leq q \leq 11$, construct a chain of $p$ copies of $G_{7}$, to which we add either a chain with $q$ vertices if $0 \leq q \leq 6$, or a cycle of order $q$ otherwise. Call the resulting graph $G^{\prime}$.

Clearly, $G^{\prime}$ is planar, of girth 7 , and it can be seen that :

- $\left|\bar{V}\left(G^{\prime}\right)\right| \geq \frac{N-q}{6}$ if $0 \leq q \leq 6$;

- $\left|\bar{V}\left(G^{\prime}\right)\right| \geq \frac{N-q}{6}+1$ if $7 \leq q \leq 11$.

In all the cases, we see that $\left|\bar{V}\left(G^{\prime}\right)\right| \geq \frac{N}{6}-1$.

\subsection{Outerplanar Graphs and 1-Planar Graphs}

An outerplanar graph $G$ is a graph that can be drawn on the plane in such a way that it is planar and all its vertices are lying on one face. The following is a well-known result, that can be found for instance in [18].

Theorem 13 For any outerplanar graph $G, a(G) \leq 3$.

By application of Lemma 1 on the above result, we get the following proposition.

Proposition 14 (MFVS in Outerplanar Graphs - Upper bound) For any outerplanar graph $G$ of $\operatorname{order} N,|\bar{V}(G)| \leq \frac{N}{3}$.

Moreover, it is possible to show that the general lower bound given in Proposition 14 above is tight.

Proposition 15 (MFVS in Outerplanar Graphs - Lower bound) For any integer $N \geq 3$, there exists an outerplanar graph $G$ of order $N$, such that $|\bar{V}(G)|=\left\lfloor\frac{N}{3}\right\rfloor$.

Proof: Consider the graph constructed in proof of Proposition 3. This graph is also an outerplanar graph, thus we can conclude directly that $|\bar{V}(G)|=\left\lfloor\frac{N}{3}\right\rfloor$.

A graph $G$ is said to be 1-planar if it can be drawn in the plane in such a way that every edge crosses at most one other edge. We have the following result, proved in [4].

Theorem 16 [4] For any 1-planar graph $G, a(G) \leq 20$.

By application of Lemma 1 on the above results, we get the following proposition.

Proposition 17 (MFVS in 1-Planar Graphs - Upper bound) For any 1-planar graph $G$ of $\operatorname{order} N,|\bar{V}(G)| \leq \frac{9 N}{10}$.

Proposition 18 (MFVS in 1-Planar Graphs - Lower bound) For any arbitrary $N \geq 8$, there exists a 1-planar graph $G$ of order $N$ such that $|\bar{V}(G)| \geq \frac{5 N}{8}-2$.

Proof : Consider the hypercube of dimension $3, H_{3}$, to which all the diagonals have been added. Call this graph $H^{\prime} . H^{\prime}$ is 1-planar (cf. Figure 4), and has been shown to satisfy $a\left(H_{1}\right)=7$ [4], and thus $\left|\bar{V}\left(H^{\prime}\right)\right| \leq 5$ by Lemma 1 . However, $\left|\bar{V}\left(H^{\prime}\right)\right| \geq 5$ by Lemma 2 . Thus $\left|\bar{V}\left(H^{\prime}\right)\right|=5$. Now, for any $N \geq 8, N=8 p+q(p \geq 1$ and $0 \leq q \leq 7)$, we take $p$ copies of $H^{\prime}$ (call them $\left.H_{1}^{\prime}, H_{2}^{\prime} \ldots H_{p}^{\prime}\right)$, and connect exactly one vertex of $H_{i}^{\prime}$ to a vertex of $H_{i+1}^{\prime}, 1 \leq i \leq p-1$. There remains $q$ vertices 
to add to this graph : if $1 \leq q \leq 6$, then take a copy of $K_{q}$ and connect any of its vertices to a vertex of $H_{p}^{\prime}$ (it is easy to see that $K_{q}$ is 1 -planar when $1 \leq q \leq 6$ ). If $q=7$, take a copy of $K_{6}$ and connect it as described above, and add a new vertex connected by a pendent edge to the construction. Call this new graph $G$. It can be easily seen that for any $N \geq 8, G$ is 1-planar. Moreover, for each copy of $H^{\prime}$ it contains, the cardinality of a MFVS must be increased by 5 . Finally, the $q$ "last" vertices we have added will increase the cardinality of a MFVS by $q-2$ if $3 \leq q \leq 6$, and $q-3$ if $q=7$.

Globally, we have $|\bar{V}(G)| \geq 5 p+f(q)$, where $f(q)=0$ if $0 \leq q \leq 2, f(q)=q-2$ if $3 \leq q \leq 6$ and $f(q)=4$ if $q=7$. In all the cases, we can see that $|\bar{V}(G)| \geq \frac{5 N}{8}-2$.

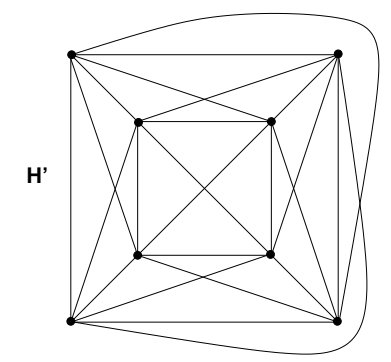

Figure 4: Graph $H^{\prime}$ is 1-planar

\section{$3.5 k$-Trees}

We recall the definition of a $k$-tree [6] :

(a) A clique with $k$-vertices is a $k$-tree.

(b) If $T=(V, E)$ is a $k$-tree and $C$ is a clique of $T$ with $k$ vertices and $x \notin V$, then $T^{\prime}=$ $(V \cup\{x\}, E \cup\{(c, x): c \in C\})$ is a $k$-tree.

It is well-known that 1-trees are trees, and that outerplnar graphs are partial 2-trees. We now prove the following simple observation.

Observation 1 For any $k$-tree $G_{k}$ of order $N \geq k+1, a\left(G_{k}\right)=k+1$.

Proof : By definition of a $k$-tree $G_{k}$, we know that at least $k+1$ colors are necessary to acyclically color any $G_{k}$ of order $N \geq k+1$, since in that case $K_{k+1}$ is a subgraph of $G_{k}$. Moreover, we can show that $a\left(G_{k}\right) \leq k+1$ for any $k \geq 1$ using the following coloring : first, color the vertices of $K_{k}$ with $k$ pairwise different colors. Each time a new vertex is added (with edges $\left(u, u_{i}\right)$, where $u_{i}, 1 \leq i \leq k$ are vertices of a complete graph $K_{k}$ ), use the only color among $1,2 \ldots k+1$ that is not used by any of the $u_{i}$. This coloring is clearly proper (no two neighbors are assigned the same color), and it is also acyclic. Indeed, no bicolored cycle can go through $u$, because for any pair $u_{p}, u_{q}$ of neighbors of $u, u_{p}$ and $u_{q}$ are assigned pairwise different colors. This shows that for any $k$-tree $G_{k}, a\left(G_{k}\right) \leq k+1$. Altogether, we then get the result.

By the above observation anf by appliaction of Lemma 1, we get the following result.

Proposition 19 (MFVS in $k$-Trees - Upper bound) For any $k$-tree $G_{k}$ of order $N$, $\left|\bar{V}\left(G_{k}\right)\right| \leq \frac{k-1}{k+1} \cdot N$.

Remark 1 We note that in the very special case where $k=1$, the result is optimal since 1 -trees are trees, and thus $\left|\bar{V}\left(G_{1}\right)\right|=0$. 
Proposition 20 (MFVS in $k$-Trees - Lower bound) For any arbitrary $k \geq 2$ and $N \geq k+1$, there exists a $k$-tree $G_{k}$ of order $N$, such that $\left|\bar{V}\left(G_{k}\right)\right| \geq \frac{k-1}{k+1} \cdot N-2$.

Proof : For any $k \geq 2$ and any $N \geq k+1$, let $N=p \cdot(k+1)+q$, where $p \geq 1$ and $0 \leq q \leq k$. We will construct the $k$-tree $G_{k}$ using the following method : start by a copy of the complete graph $K_{k+1}$, and call its vertices $u_{1}, u_{2}, \ldots u_{k+1}$. Now, for every $k+2 \leq i \leq N$, connect vertex $u_{i}$ with all vertices $u_{i-1}, u_{i-2}, \ldots u_{i-k}$. An example of this construction is shown in Figure 5 in the case $k=3$ and $N=10$. This construction clearly gives a $k$-tree, because one can see (by induction) that for any $k+2 \leq i \leq N$, the graph induced by vertices $u_{i-1}, u_{i-2}, \ldots u_{i-k}$ is isomorphic to the complete graph $K_{k}$.

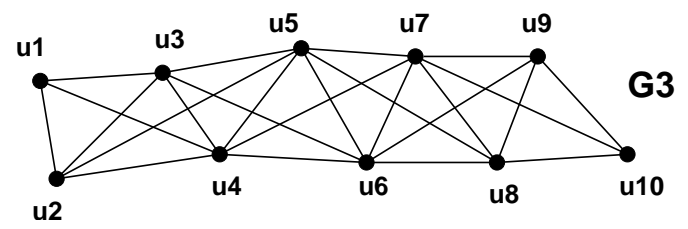

Figure 5: The 3-tree $G_{3}$ of order $N=10$

In order to prove that $\left|\bar{V}\left(G_{k}\right)\right| \geq \frac{k-1}{k+1} \cdot N-2$, it suffices to note that for any integer $0 \leq l \leq p-1$, the set of vertices $V_{l}=\left\{u_{l(k+1)+1}, u_{l(k+1)+2}, \ldots u_{(l+1)(k+1)}\right\}$, induces a graph that is isomorphic to the complete graph $K_{k+1}$. However, we know that for any $m \geq 2,\left|\bar{V}\left(K_{m}\right)\right|=m-2$. Thus, for any set $V_{l}, 0 \leq l \leq p-1$, it is necessary to remove at least $k-1$ vertices in $G_{k}$ in order to get an acyclic graph. However, if $q \geq 3$, this is not sufficient. Indeed, let $V^{\prime}=$ $\left\{u_{p(k+1)+1}, u_{p(k+1)+2}, \ldots u_{p(k+1)+q}\right\}$. The graph induced by the vertices of $V^{\prime}$ is also isomorphic to a complete graph, more precisely to $K_{q}$. Thus it is necessary to remove also $q-2$ vertices to $G_{k}$ in order to get an acyclic graph. We then distinguish two cases :

- $q \in\{0,1,2\}$. In that case, $\left|\bar{V}\left(G_{k}\right)\right| \geq(k-1) \cdot p$. However, $N=p \cdot(k+1)+q$, thus $p=\frac{N-q}{k+1}$ and consequently $\left|\bar{V}\left(G_{k}\right)\right| \geq \frac{k-1}{k+1} \cdot N-\frac{k-1}{k+1} \cdot q$, which satisfies the inequality of Proposition 20 since $k \geq 2$ and $0 \leq q \leq 2$.

- $3 \leq q \leq k$. In that case, $\left|\bar{V}\left(G_{k}\right)\right| \geq(k-1) \cdot p+(q-2)$. Since $N=p \cdot(k+1)+q$, we then get $\left|\bar{V}\left(G_{k}\right)\right| \geq \frac{k-1}{k+1} \cdot N+\frac{2 q}{k+1}-2$. However, since $3 \leq q \leq k$, it can be seen that $\frac{2 q}{k+1}-2$ is always greater than or equal to -2 .

In all the cases, we have shown that for any $k \geq 2$ and $N \geq k+1$, there exists a $G_{k}$ for which we have $\left|\bar{V}\left(G_{k}\right)\right| \geq \frac{k-1}{k+1} \cdot N-2$.

Remark 2 We note that in the case $k=2$, it is possible to refine this lower bound (more precisely the additive constant between the lower and upper bound is no more than 1). Indeed, the construction of $G_{2}$ in proof of Proposition 20 above in the case $k=2$ yields a graph with $N$ vertices, $2 N-3$ edges and of maximum degree 4. Using the lower bound of Lemma 3, we then get $\left|\bar{V}\left(G_{2}\right)\right| \geq \frac{N-2}{3}$.

\section{Conclusion}

In this paper, our main contribution is to make an interesting connection between the acyclic chromatic number of a graph $G$ and the cardinality of its MFVS. This connection shows that graphs "live" on a special region of the 2- $D$ space < acyclic chromatic number, MFVS cardinality $>$. This region delimited by Lemmas 1 and 2 is shown to be optimal since we have graph families on the borders (outerplanar graphs and $k$-trees for the upper border, complete $k$-partite graphs for the lower one).

We then have exploited already known deep results on the acyclic coloring of large families 
of graphs to get new and good approximations on the MFVS cardinality for graphs belonging to large families, such as graphs of maximum degree 3, of maximum degree 4, planar graphs, planar graphs with given girth, 1-planar graphs, showing that the acyclic coloring approach gives fruitful results for the MFVS problem. We note that, conversely, getting relevant results concerning the acyclic chromatic number from known MFVS cardinality results remains an open question (the only result being Lemma 2 ).

Tables 1 and 2 summarize the results presented here, as well some other results that we have referred to in this paper (i.e. complete graphs, complete $k$-partite graphs). The numbers that appear between parentheses refer to results coming from this paper. The ones that appear between brackets are citations from which those results come from. In Table 1, the numbers displayed in the column "Ratio" give the multiplicative ratio that exists between the lower and upper bounds for the MFVS cardinality. When this ratio is equal to 1 (thus leading to an optimal result), it is given in bold characters.

\begin{tabular}{||c||c||c|c|c||}
\hline \hline \multicolumn{1}{||c||}{} & \multicolumn{1}{|c||}{} & \multicolumn{2}{c||}{ MFVS Cardinality } & Ratio \\
\hline Family $\mathcal{F}$ & $|V|$ & Lower Bound & Upper Bound & \\
\hline \hline Complete graphs $K_{N}$ & $N$ & \multicolumn{2}{c||}{$N-2$} & $\mathbf{1}$ \\
\hline $\begin{array}{c}\text { Complete } k \text {-partite } \\
\text { graphs } K_{n_{1}, n_{2}, \ldots n_{k}}\end{array}$ & $\sum_{i=1}^{k} n_{i}$ & \multicolumn{2}{c||}{$\sum_{i=1}^{k} n_{i}-\max \left(n_{i}\right)-1$} & $\mathbf{1}$ \\
\hline Maximum Degree 3 & $N$ & $\frac{N}{3}$ (Prop. 3) & $\frac{N}{2}$ (Prop. 2) & 1.5 \\
\hline Maximum Degree 4 & $N$ & $2 \cdot \frac{N}{4} \mid$ (Prop. 6) & $\frac{3 N}{5}$ (Prop. 5) & 1.2 \\
\hline Planar & $N$ & $\frac{N}{2} \mid$ (Prop. 8) & $\frac{3 N}{5}$ (Prop. 7) & 1.2 \\
\hline Planar with girth $=5,6$ & $N$ & $\frac{3 N}{10}-2$ (Prop. 11) & $\frac{N}{2}$ (Prop. 10a) & 1.67 \\
\hline Planar with girth $\geq 7$ & $N$ & $\frac{N}{6}-1$ (Prop. 12) & $\frac{N}{3}$ (Prop. 10b) & 2 \\
\hline 1-Planar Graphs & $N$ & $\frac{5 N}{8}-2$ (Prop. 18) & $\frac{9 N}{10}$ (Prop. 17) & 1.44 \\
\hline Outerplanar Graphs & $N$ & \multicolumn{2}{|c|}{$\left.\frac{N}{3}\right\rfloor$ (Prop. 14 and 15) } & $\mathbf{1}$ \\
\hline 2-Trees & $N \geq 3$ & $\frac{N-2}{3}$ (Rem. 2) & $\frac{N}{3}$ (Prop. 19) & $\mathbf{1}$ \\
\hline$k$-Trees, $k \geq 3$ & $N \geq k+1$ & $\frac{k-1}{k+1} \cdot N-2$ (Prop. 20) & $\frac{k-1}{k+1} \cdot N$ (Prop. 19) & $\mathbf{1}$ \\
\hline \hline
\end{tabular}

Table 1: MFVS cardinality of some families $\mathcal{F}$ of graphs

\begin{tabular}{|c|c|c|c|}
\hline & & \multicolumn{2}{|c|}{ Acyclic Chromatic Number } \\
\hline Family $\mathcal{F}$ & $|V|$ & Lower Bound & Upper Bound \\
\hline Complete graphs $K_{N}$ & $\bar{N} N$ & \multicolumn{2}{|c|}{$N$} \\
\hline Complete $k$-partite graphs $K_{n_{1}, n_{2}, \ldots n_{k}}$ & $\overline{\sum_{i=1}^{k} n_{i}}$ & \multicolumn{2}{|c|}{$\sum_{i=1}^{k} n_{i}-\max \left(n_{i}\right)+1$} \\
\hline Maximum Degree 3 & $N$ & \multicolumn{2}{|c|}{$4[13]$} \\
\hline Maximum Degree 4 & $N$ & \multicolumn{2}{|c|}{$5[8]$} \\
\hline Planar & $N$ & \multicolumn{2}{|c|}{$5[3]$} \\
\hline Planar with girth $=5,6$ & $N$ & \multicolumn{2}{|c|}{$4[5]$} \\
\hline Planar with girth $\geq 7$ & $N$ & \multicolumn{2}{|c|}{$3[5]$} \\
\hline 1-Planar Graphs & $\bar{N}$ & $7[4]$ & $20[4]$ \\
\hline Outerplanar Graphs & $N$ & \multicolumn{2}{|c|}{$3[18]$} \\
\hline 2 -Trees & $N \geq 3$ & \multicolumn{2}{|c|}{3 (Obs. 1) } \\
\hline$k$-Trees, $k \geq 3$ & $N \geq k+1$ & \multicolumn{2}{|c|}{$k+1$ (Obs. 1) } \\
\hline
\end{tabular}

Table 2: Acyclic chromatic number of some families $\mathcal{F}$ of graphs 


\section{Acknowledgements}

The authors would like to thank the referees for their remarks, which helped improve the paper.

\section{References}

[1] V. Bafna, P. Berman, and T. Fujito. Constant ratio approximations of the weighted feedback vertex set problem for undirected graphs. In Proc. ISAAC'95, Algorithms and Computation, volume 1004, pages 142-151, 1995. Lectures Notes in Computer Science, Springer-Verlag, Berlin.

[2] L. W. Beineke and R.C. Vandell. Decycling graphs. J. Graph Theory, 25:59-77, 1997.

[3] O.V. Borodin. On acyclic colorings of planar graphs. Discrete Mathematics, 25:211-236, 1979.

[4] O.V. Borodin, A.V. Kostochka, A. Raspaud, and E. Sopena. Acyclic colouring of 1-planar graphs. Discrete Applied Mathematics, 114(1-3):29-41, 2001.

[5] O.V. Borodin, A.V. Kostochka, and D.R. Woodall. Acyclic colourings of planar graphs with large girth. J. London Math. Soc., 60 (2):344-352, 1999.

[6] A. Brandstädt, V.B. Le, and J.P. Spinrad. Graph Classes : A survey. SIAM Monographs on D.M. and Applications, 1999.

[7] A. Brandstädt. On improved time bounds for permutation graphs problem. In 18th International Workshop on Graph-Theoretic Concepts in Computer Science (WG '92), volume 657 of Lecture Notes in Computer Science, pages 1-10. Springer-Verlag, 1992.

[8] M.I. Burstein. Every 4-valent graph has an acyclic 5 coloring (in russian). Soobšč. Akad. Nauk Gruzin, SSR 93:21-24, 1979.

[9] I. Caragiannis, C. Kaklamanis, and P. Kanellopoulos. New bounds on the size of the minimum feedback vertex set in meshes and butterflies. In Proc. 8th International Colloquium on Structural Information and Communication Complexity (SIROCCO '01), volume 11, pages $77-88,2001$.

[10] S.R. Coorg and C.P. Rangan. Feedback vertex set on cocomparability graphs. Networks, 26:101-111, 1995.

[11] P. Festa, P.M. Pardalos, and M.G.C. Resende. Feedback set problems. Handbook of Combinatorial Optimization, Supplement Vol. A, (Eds: DingZhu Du and Panos M. Pardalos), Kluwer Academic Publishers, pages 209-258, 1999.

[12] R. Focardi, F.L. Luccio, and D. Peleg. Feedback vertex set in hypercubes. Information Processing Letters, 76:1-5, 2000.

[13] B. Grünbaum. Acyclic colorings of planar graphs. Israel J. Math., 14(3):390-408, 1973.

[14] J. Kleinberg and A. Kumar. Wavelength conversion in optical networks. J. of Algorithms, $38(1): 25-50,2001$.

[15] C.L. Lu and C.Y. Tang. A linear-time algorithm for the weighted feedback vertex problem on interval graphs. Information Processing Letters, 61:107-111, 1997.

[16] F.L. Luccio. Almost exact minimum feedback vertex set in meshes and butterflies. Information Processing Letters, 66:59-64, 1998. 
[17] D. Peleg. Local majority voting, small coalitions and controlling monopolies in graphs : A review. In 3rd International Colloquium on Structural Information and Communication Complexity (SIROCCO '96), Proceedings in Informatics, pages 152-169. Carleton Scientific, 1996.

[18] E. Sopena. The chromatic number of oriented graphs. Mathematical Notes, 25:191-205, 1997.

[19] O. Togni. Placement de convertisseurs de longueurs d'ondes dans les réseaux optiques. In 2èmes Rencontres Francophones sur les Aspects Algorithmiques des Télécommunications (AlgoTel 2000), pages 35-40. INRIA, 2000. 